\title{
HIV replication and evolution in patients on highly active antiretroviral therapy Robert F Siliciano*
}

\begin{abstract}
Address: Johns Hopkins University School of Medicine and Howard Hughes Medical Institute, Baltimore, Maryland, USA
\end{abstract}
* Corresponding author

from 2006 International Meeting of The Institute of Human Virology

Baltimore, USA. 17-21 November, 2006

Published: 2I December 2006

Retrovirology 2006, 3(SuppI I):SI I doi:I0.II86/I742-4690-3-SI-SI I

(C) 2006 Siliciano; licensee BioMed Central Ltd.

Treatment of HIV-1 infection with highly active antiretroviral therapy (HAART) reduces viremia to below the detection limit of ultrasensitive clinical assays. However, HIV-1 persists in resting CD4+ T cells and possibly other reservoirs. In patients on HAART, HIV-1 persistence is evidenced not only by the latent reservoir in resting CD4+ T cells but also by free virus in the plasma. Given the short half-life of free virus, this residual viremia indicates active virus production. This virus production may reflect lowlevel ongoing replication that continues despite HAART and/or release of virus from latently infected cells that become activated or from other stable cellular reservoirs. With respect to the issue of drug resistance, it is of particular importance to determine whether ongoing replication continues on HAART because the evolution of resistance can only take place if there are new cycles of replication. The direct characterization of residual viremia provides a window into this state of virologic suppression and a means for determining the importance of different mechanisms of viral persistence. Characterization of this residual viremia has been limited because of the technical difficulties involved in the analysis of extremely low numbers of viral RNA templates. To obtain sufficient numbers of independent viral clones from the plasma of patients on HAART, we have carried out intensive sampling in a series of patients and analyzed plasma virus genotypes with a sensitive RT-PCR method. Viral variants in the plasma were compared to viruses in the latent reservoir. The results provided evidence that in some patients on HAART, much of the residual viremia is due to continued production of a small number of viral clones over prolonged periods without evident sequence change by cells that are not well represented in the circulation. The static nature of the residual viremia is not consistent with ongoing viral replication. These results have implications for understanding HIV-1 persistence and treatment failure. 